\title{
Surface Studies
}

\section{of \\ MBE-Grown Semiconductor Films}

\author{
P.K. Larsen, Eindhoven and B.A. Joyce, Redhill \\ (Philips' Research Laboratories)
}

The past decade has seen rapid progress in two apparently unrelated fields. The first, surface physics, has established the possibility of determining the electronic and crystallographic structure of surfaces as well as their chemical composition. The second, molecular beam epitaxy (MBE), is a thin film preparation technique, applied especially to semiconductors, which makes possible the preparation of structures having highly controlled characteristics le.g. chemical composition, dopant concentration and profile, thickness). The combination of MBE with the techniques used in surface physics, all of which are based on operation in ultra high vacuum (UHV), has opened up new possibilities of semiconductor surface studies. MBE also has a much wider application than this, particularly in the burgeoning field of low-dimension solids ${ }^{1}$ ) - quantum well structures and periodic super lattices - for which it is one of the two enabling technologies, the other being organo-metallic vapour phase epitaxy which is outside the scope of this article.

\section{Molecular Beam Epitaxy}

Almost all of the earlier work on semiconductor surfaces relied for sample preparation on either cleavage or thermal annealing, possibly combined with ion bombardment. Cleavage restricts measurements to a single plane, however. Ion bombardment and annealing are not appropriate to compound semiconductors, where controlled surface stoichiometry is crucial. By using MBE these limitations are removed and a wide range of semiconductor surfaces can be prepared in-situ with a high degree of stoichiometric control and crystallographic perfection. The method uses directed neutral thermal atomic and molecular beams, which impinge onto a heated single-crystal substrate maintained under UHV conditions. Although the earliest work on MBE was concerned with $\mathrm{Si}$, most recent developments have concentrated on III-V semiconductors. The growth process for (001) oriented GaAs is illustrated in Fig. 1. The beams which are generated by
Knudsen effusion cells, for Group III elements ( $\mathrm{Al}, \mathrm{Ga}, \mathrm{In}$ ) are composed of atoms, whereas Group V elements ( $P$, $\mathrm{As}, \mathrm{Sb})$ form either tetramers or dimers. Growth rates are typically one monolayer (ML) per second and film thickness can be controlled down to atomic dimensions. Equivalent control of the interface abruptness between two different semiconductor layers can also be achieved.

The (001)-oriented crystal illustrated in Fig. 1 is composed of alternating layers of covalently bonded $\mathrm{Ga}$ and $\mathrm{As}$ atoms. This implies that the surface can be terminated by either $\mathrm{Ga}$, As, or a mixture of both types of atom. With MBE growth it is possible to arrange the conditions so as to specify the surface stoichiometry (i.e. Ga to As surface concentration ratio) and so the crystallographic surface structure. In comparison with the bulk atom positions in general with semiconductors, there is a reduction in symmetry at the surface due to atomic rearrangements and the surface unit cell has a larger periodicity than that of the ideal bulk terminated lattice. This is known as a reconstructed surface and the new primitive cell is expressed in terms of the dimensions of the unreconstructed unit cell (the so-called $1 \times 1$ structure). For example, the (001) surface of $\mathrm{GaAs}$ has several reconstructions of which the most stable is the $2 \times 4$ structure. This means that along two orthogonal $\langle 110\rangle$ directions there is a twofold and a fourfold increase in the periodicity, respectively. (A particular example of such a structure is shown in Fig. 7.)

\section{Electron Diffraction and Surface Crystallography}

Surface crystallography is most conveniently monitored by electron diffraction techniques. Low-energy electron diffraction (LEED), which uses a backscattering geometry, has been extensively used for work on cleaved surfaces, but it is not really suitable in combination with MBE. For this, the forward scattering geometry of reflection high-energy electron diffraction (RHEED) is more appropriate, since the electron beam is at extreme grazing incidence (Fig. 2) whereas the molecular beams impinge almost normally on the substrate. Although the same basic information is obtained from both methods, a combination of RHEED and MBE enables the surface crystallography to be monitored even under dynamic conditions when the surface is growing.

As an example of the information which can be obtained from RHEED we consider the GaAs (100) surface. A number of different reconstructions have been observed, of which the most important in order of increasing As surface population are $4 \times 2,4 \times 6,1 \times 6,2 \times 4$ and the centred $4 \times 4$ (referred to as $c$ $(4 \times 4)$. Figure 3 shows the RHEED patterns in two-orthogonal $\langle 110\rangle$ azimuths from a $2 \times 4$ reconstructed surface, one with a twofold and the other

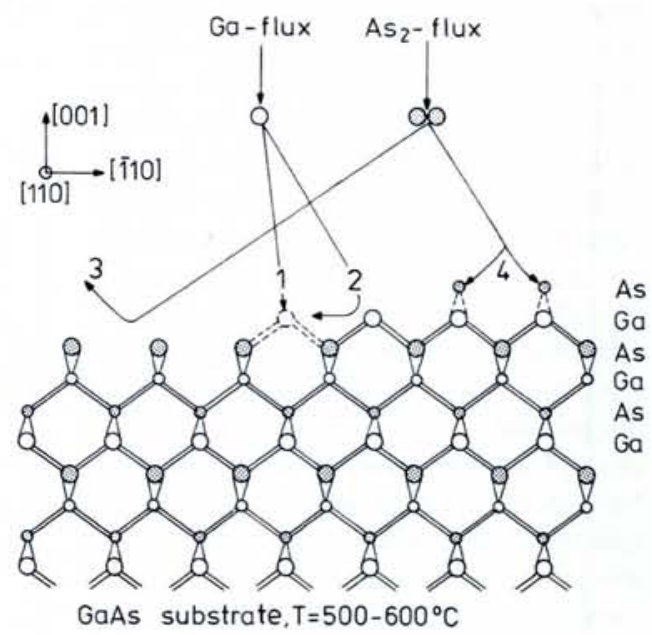

GaAs substrate, $T=500-600^{\circ} \mathrm{C}$
Fig. 1 - Schematic growth model for GaAs (001). Incident $\mathrm{Ga}$ atoms can either bond directly to As-substrate atoms (1) or first diffuse over the surface (2). Arriving $\mathrm{As}_{2}$ molecules desorb from As-terminated sites (3) or dissociatively chemisorb on $\mathrm{Ga}$ atoms (4). 


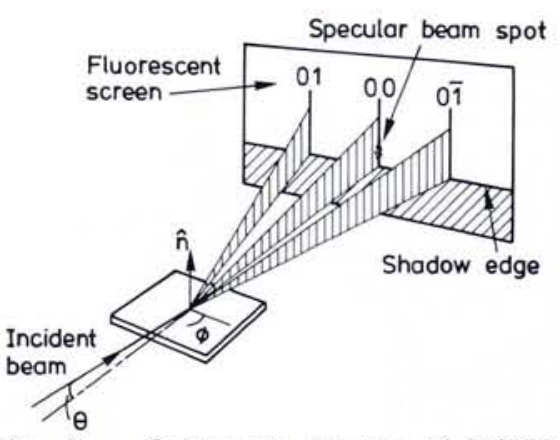

Fig. 2 - Schematic diagram of RHEED geometry showing the incident beam at an angle $\theta$ to the surface plane. The scattered beam is characterised by sets of streaks normal to the shadow edge.

with a fourfold increase in periodicity. In addition to establishing the surface geometry, the RHEED pattern can also be used to evaluate the surface morphology (surface steps, facets, antisite disorder, etc.) ${ }^{2}$ ).

The compatibility of RHEED and MBE has recently been exploited to study the dynamics of epitaxial thin-film growth by monitoring temporal variations in the intensity of any feature in the RHEED pattern. It has been found that damped oscillations occur immediately after the initiation of growth. A typical example is shown in Fig. 4. The period of oscillation corresponds exactly to the growth of a single monolayer, i.e. a complete layer of $\mathrm{Ga}$ and $\mathrm{As}$ atoms. This provides an absolute and continuous growth-rate monitor with atomic layer precision. A particularly important application is the intum wells (thin GaAs layers between $\mathrm{Ga}_{x} \mathrm{Al}_{1-x} \mathrm{As}$ ) and this is the most critical parameter in defining energy levels of the confined electrons. The observation of oscillatory effects in thin-film growth layer-by-layer growth process, i.e. twodimensional nucleation. It is assumed that the equilibrium surface is smooth and strongly reflective to electrons so situ determination of the width of quanstudies is usually associated with a

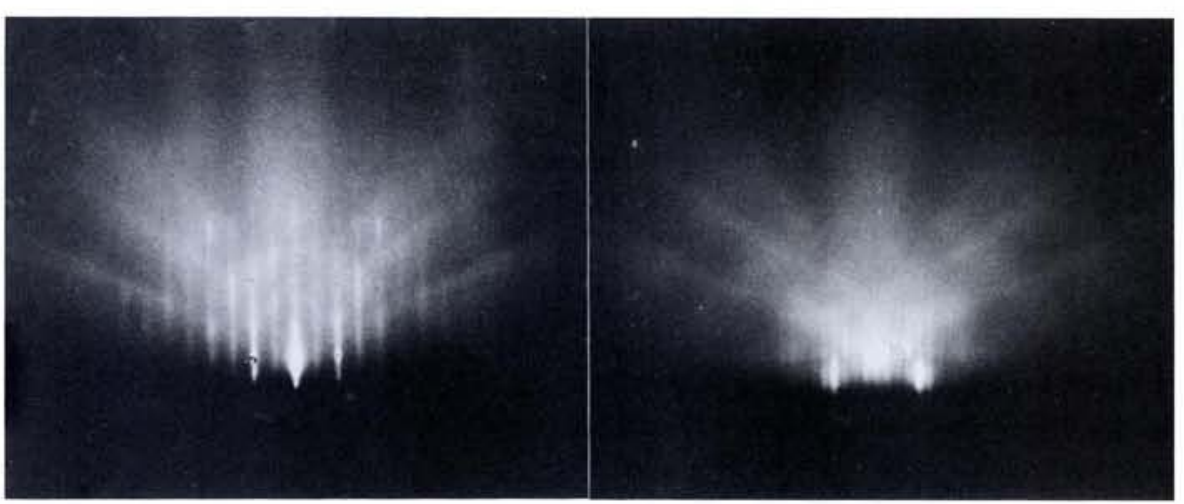

Fig. 3-RHEED patterns from two orthogonal <110> azimuths of the GaAs (001) - $2 \times 4$ reconstructed surface. that the specular beam intensity is high. As growth commences, two-dimensional clusters are formed randomly on the surface leading to a decrease in intensity; the maximum roughening (i.e. minimum intensity) occurs at half layer coverage. The oscillations are damped because the ideal layer-by-layer model is not completely followed and the surface becomes statistically distributed over several incomplete layers. Detailed analysis of the intensity requires the development of a dynamical theory (i.e. multiple scattering theory) from a randomly stepped surface which is not yet available. In the near future we may expect rapid progress in our knowledge of thinfilm growth dynamics from the application of this technique.

\section{Electronic Surface Structure}

The surface electronic structure is directly related to the surface crystallography and therefore, in principle, each reconstruction results in a surface with a specific electronic structure. Angleresolved photo-emission spectroscopy (ARPES) using monochromatized synchrotron radiation provides a powerful method for determining surface and bulk electronic structure, together with chemical bonding information ${ }^{3}$ ).

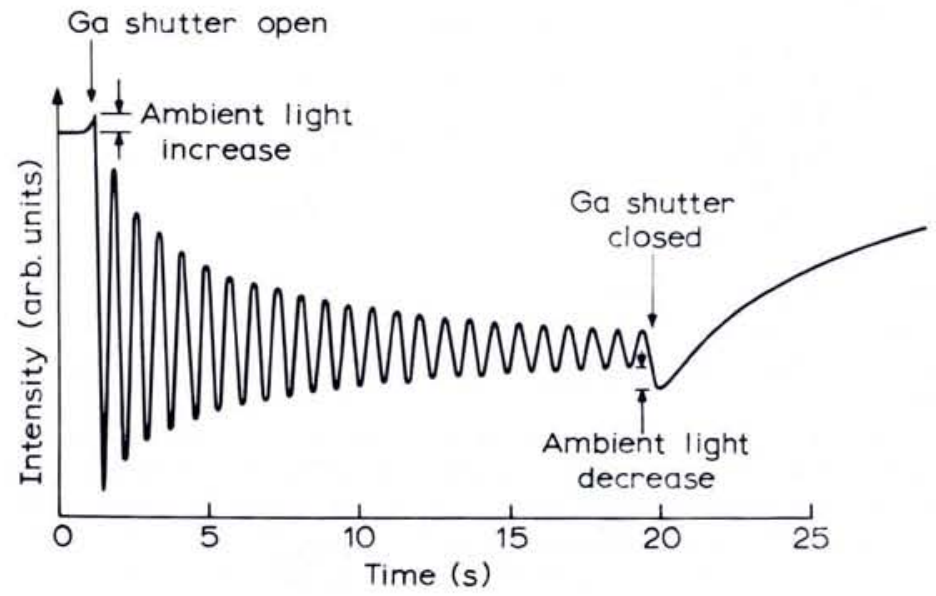

To illustrate the photo-emission technique, we show in Fig. 5 a schematic energy level diagram of a heteropolar semiconductor. The absorption of photons of energy $h v$ leads to (direct) optical transitions (e.g. transition no. 1) from an initial state (of energy $E_{i}$ ) to a final state $\left(E_{t}\right)$. Close to the surface photo-excited electrons may escape into the vacuum without being inelastically scattered and in the ARPES measurements, their kinetic energies and directions are determined which provides information on the initial states. The basis of ARPES relates directly to the conservation of the surface parallel component of the wave vector of the photo-electron when it crosses the surface on its way into the vacuum, i.e. $\vec{k}_{\|}=\vec{k}_{\| \text {vac }}+\vec{g}_{\|}$. Here, $\vec{k}$ and $\vec{k}_{\text {vac }}$ denote the wave vector in the solid and in vacuum, respectively, and $\vec{g}_{\|}$is a vector of the reciprocal surface lattice. From $E_{\text {kin, }}$ the polar angle $\theta$ between the surface normal and the electron trajectory in vacuum, and the azimuth angle $\varphi$ both the initial state energy $E_{i}$ and the wave vector $\vec{k}_{/}$can be directly determined, i.e. ARPES measurements provide a determination of two-dimensional band structures $E_{\mathrm{i}}=E_{\mathrm{i}}\left(\vec{k}_{\mu}\right)$.

In Fig. 5 two transitions from initial states in the valence band region are indicated. No. 1 is from a bulk valence band state which in general depends on $\left.\vec{k}: E_{i}=E_{i}(\vec{k})=E_{i} \vec{k}, \vec{k}_{\Downarrow}\right)$. At the surface and also at semiconductor-semiconductor and semiconductor-metal interfaces the energy states are modified and localized surface (or interface) states appear giving rise to transition no. 2, which by nature is two-dimensional, $E_{i}=E_{i}\left(\vec{k}_{\ell}\right)$. (Similarly, the different chemical environment at the surface compared to the

Fig. 4 - Intensity oscillations of the specular beam in the RHEED pattern from a GaAs (001) - $2 \times 4$ reconstructed surface, [110] azimuth. The period exactly corresponds to the growth of a single $\mathrm{Ga}+$ As layer and the amplitude gradually decreases (Neave J.H. et al., Appl. Phys. A31 (1983) '). 


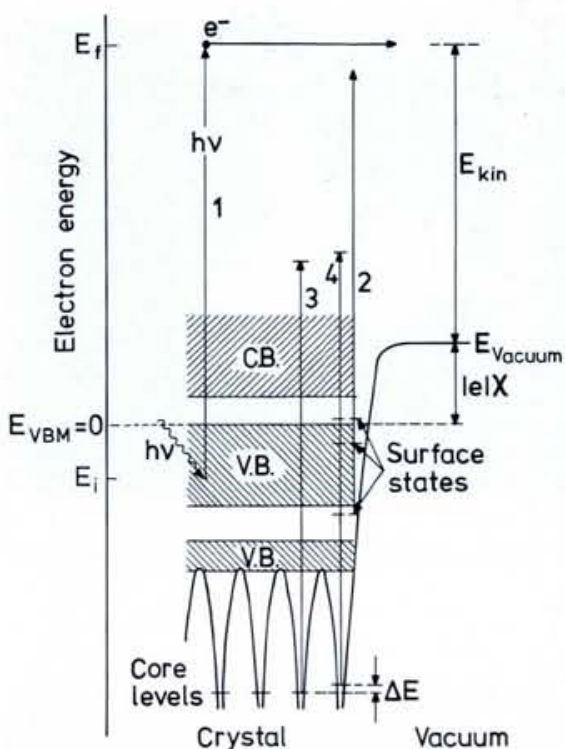

Fig. 5-Schematic energy level diagram for a heteropolar semiconductor illustrating the photo-emission process. The valence band maximum $\left(E_{V B M}\right)$ is taken as the zero of energy and $\mid e / \chi$ refers the vacuum potential to this.

bulk may cause an energy shift $(\triangle E)$ in the core level binding energy and therefore different core level transitions, nos. 3 and 4 .) We are here primarily concerned with the surface electronic structure, not the bulk, but both types of transition are manifest in the photo-emission spectra and have to be distinguished. The use of MBE to produce different surface structures of the same bulk crystal provides a unique opportunity of making this distinction. This is demonstrated in Fig. 6, which shows three spectra from different $\mathrm{GaAs}$ (001) surface structures measured under identical conditions using synchrotron radiation from the ACO synchrotron storage rings, LURE, Orsay. For initial energies $E_{\mathrm{i}}<-2.7 \mathrm{eV}$, the spectra are qualitatively very similar and this region is dominated by bulk emission. The peaks $X_{3}$ and $\Sigma_{1}^{\min }$ occur at the energy of the $X_{3}$ and $\Sigma_{1}^{\text {min }}$ band structure extrema and are observed over a wide range of experimental conditions because of the flatness of the energy bands and the resulting high density of states. The peak labelled 1 represents a transition from a valence band state. The final state for such bulk transitions at sufficiently high photon energies $(h v \geq$ $20 \mathrm{eV}$ ) is found to be free-electron-like for GaAs and as a result the threedimensional bulk band structure can be directly determined ${ }^{3}$ ).

When we consider energies higher than $-2.7 \mathrm{eV}$, however, there are few similarities in the spectra shown in Fig. 6 . Energy positions of peaks and shoulders from the $2 \times 4$ and $\mathrm{c}(4 \times 4)$ recons-
Fig. 6-Angle-resolved photo-electron spectra taken at a polar angle of 270 in the [010] azimuth for three GaAs (001) surface reconstructions (Larsen P.K. et al., Phys. Rev. B 26 (1982) $322{ }^{2}$ ).

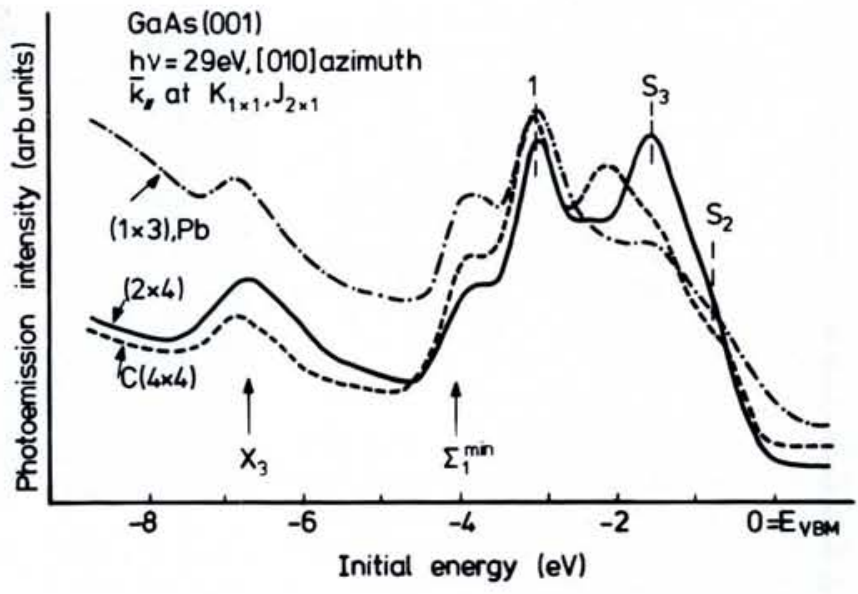

tructions are quite different, and the emission is strongly quenched in the presence of an overlayer structure of lead. This strongly indicates that the prominent emission features, e.g. $\mathrm{S}_{2}$ and $\mathrm{S}_{3}$ for the $2 \times 4$ structure, are surface state related.

Another technique used to distinguish valence band and surface states, where the tunability of synchrotron radiation plays a decisive part, relies on keeping $\vec{k}_{\|}$ at a constant value while changing the photon energy (achieved by changing both $h v$ and $\theta$ ). Dispersive peaks depend on $k$ while surface states do not. Using this procedure the states $S_{2}$ and $S_{3}$ are again identified as surface states.

Surface states in semiconductors can generally be associated with broken bonds of surface atoms and the modified bonds between these atoms and their neighbours. The creation of a (001) As terminated $\mathrm{GaAs}$ surface would

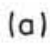

(b)

(c)

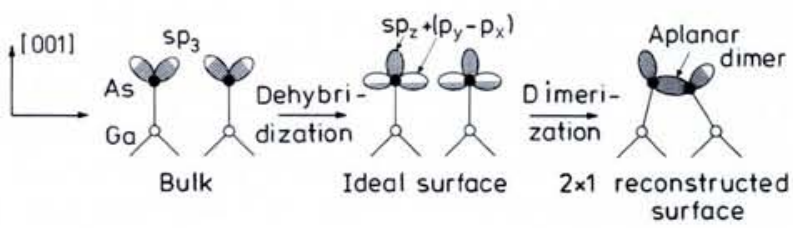

(d)

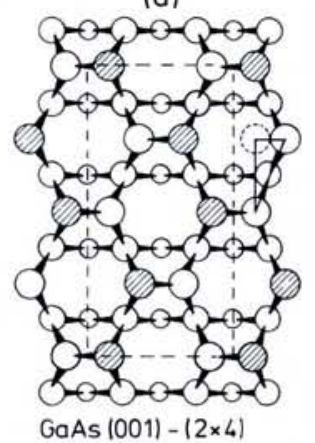

"Up"- atom of aplanar dimer

"Down"-atom of aplanar dimer

Ga-atoms in second layer

As-atoms in third layer

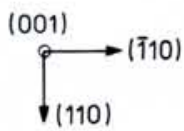

Fig. 7 - Schematic diagram of the two disrupted $s p_{3}$ lobes per surface atom on an Asterminated newly created GaAs (001) surface (a), the dehybridized dangling bond ( $s p_{z}$ ) and bridge bond $\left(p_{y}-p_{x}\right)$ on the ideal surface $(b)$, a planar dimer model for the reconstructed 2 $\times 1$ surface $(c)$, and a top view of the $2 \times 4$ full reconstructed asymmetric dimer model (d). The hatching of the lobes $(a-c)$ is an indication of the number of electrons per bond. 
Returning to the photo-emission studies we note that we are able to determine the surface electronic structure experimentally, and from intensity measurements we can establish symmetries and the orbital character of the states. These results must be compared with calculated surface energy bonds to check models. Such calculations are difficult and expensive for large surface unit cells and have so far for GaAs (001) been restricted to the non-existing, but calculable $2 \times 1$ reconstruction. There is, however, a good qualitative agreement between the measurements for the $2 \times 4$ reconstruction and the calculated surface bonds for the $2 \times 1$ dimer model illustrated in Fig. 7c. The dimer bond is found to have approximately the same binding energies $\left(-3.5 \mathrm{eV} \leq E_{\mathrm{i}} \leq-3.0\right.$ $\mathrm{eV})$ and $\vec{k}_{\mu}$-values by both calculations and measurements, while states which are associated with broken bonds and bonds to $\mathrm{Ga}$-atoms in the second layer, are found both theoretically and experimentally in the energy range $-2.5 \mathrm{eV} \leq$ $E_{\mathrm{i}} \leq 0 \mathrm{eV}$.

\section{Conclusions}

In this article we have shown how the combination of a UHV-based thin film growth technique (MBE) with ARPES and RHEED has provided unique opportunities for the study of a wide range of semiconductor surfaces and interfaces. The limitations of cleavage and ion bombardment are removed and the grown surfaces are free of damage and show precisely controlled composition. We have used the GaAs (001) - $2 \times 4$ reconstruction to illustrate the method. This surface cannot be reproducibly prepared by any other means.

In addition to evaluating the crystallographic and electronic structure of asgrown surfaces we have shown how RHEED can be used to study thin-film growth dynamics. Space does not permit the discussion of core level spectroscopy and interface studies, but they are readily incorporated into this general area, to the extent that surface and interface chemical effects are already being studied by measurements of core level binding energies and energy shifts, which can also provide information on the values of band edge discontinuities at semiconductor heterojunctions.

\section{REFERENCES}

1. Gossard A.C., Treatise on Materials Science and Technology 24 (Academic Press, New York) 1983, p. 13.

2. Joyce B.A., Neave J.H., Dobson P.J. and Larsen P.K., Phys. Rev. B29 (1984) 814.

3. Braun W. and Bradshaw A.M., Europhysics News 15 (1984) 6.

\section{The Jülich Nuclear Research Centre}

(Kernforschungsanlage Jülich)

\section{is seeking for its Solid State Research Institute}

(Institut für Festkörperforschung, IFF) a

\section{DIRECTOR}

\section{as Head of one of its experimental institutes (previously the Institute for Superconductivity and Low-temperature Physics).}

The IFF comprises seven experimental and three theoretical institutes, which carry out basic research in solid state physics, together with the necessary infrastructure. The applicant should have outstanding knowledge and experience in experimental condensed matter physics and be capable of leading a scientific institute. He should be able to complement the present activities of the IFF by a new and promising field, preferentially in the area of disorder phenomena or of phase boundaries and internal interfaces. It would be preferable if the institute would use German and international neutron sources (FRJ-2 Jülich; ILL Grenoble, later the Jülich Spallation Neutron Source, SNQ), and synchrotron sources (Hasylab, Hamburg; Bessy, Berlin; later the European Synchrotron Radiation Facility ESRF). Particular importance will be attached to a willingness to work with the other experimental and theoretical groups of the IFF. It is planned to arrange a joint appointment as a full professor (C-4) with one of the universities of North-Rhine-Westfalia.

Applications with curriculum vitae, list of publications and a short account of previous scientific activity should be sent by 15 May, 1985 to

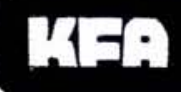

VORSTAND DER KERNFORSCHUNGSANLAGE JÜLICH GmbH

Postfach 1913

D - 5170 Jülich

\section{Council Decisions}

The EPS Council met in Berlin (West) on 22/23 March 1985 following meetings of the Executive Committee and various advisory committees, reports on which will be given in the May issue of Europhysics News. Coincidentally, the 5th EPS General Condensed Matter Physics Conference was being held at the Technical University during which generous exposure was given to EPS, and participants not yet IOMs were urged to add their weight to the Society.

Decisions taken by the Council included: the election of the Executive Committee for 1985/86 which took office immediately after the Council meeting; the changes to the Constitution and By-laws to cater more effectively for the suspension of members who do not pay their annual fees, and the provision of a reduced membership fee for young physicists (below 30 years of age) as well as retired physicists (already agreed). The relevant articles and rule are cited overleaf.

Council by a large majority also empowered the Executive Committee to raise the unit fee from the beginning of 1986 from its present value of Sw.Fr. 10. - to Sw.Fr. 11. - . The last rise took place at the beginning of 1983 and Council has in the past approved the principle that the unit fee should, in general terms, keep pace with Swiss inflation, as this represents the increase in much of the Society's expenditure. Although inflation in Switzerland tends to rise more slowly than in most European countries, the yearly rate is rarely below $3 \%$ and in 1985 has been running at nearly $10 \%$. The three-year figure, $1983-85$, is thus likely to be well over the $10 \%$ mark.

\section{Executive Committee $1985 / 86$}

President

Vice-President

Secretary

Vice-Secretary

Treasurer

Vice-Treasurer

Members
G.H. Stafford, Oxford W. Buckel, Karlsruhe W.J. Merz, Zurich

N. Kroo, Budapest D. Kuhn, Innsbruck E.W.A. Lingeman, Amsterdam

B. Dreyfus, Grenoble J.M. Gilles, Namur O.V. Lounasmaa, Espoo K.K. Rebane, Tallinn

R.A. Ricci, Padova

Outgoing members completing the maximum term of office were:

J. Kaczer, Prague

G. Preparata, Bari 